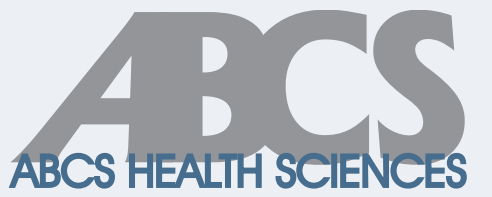

How to cite this article: Keomma, Cavalcanti Adherence in Brazil is phenomenal, but early treatment with chloroquine and hydroxychloroquine does not reduce mortality from COVID-19. ABCS Health Sci. 2021;46:e021103.

https://doi.org/10.7322/abcshs.2021175.1888

Corresponding author: Kaio Keomma Faculdade de Saúde Pública, Universidade de São Paulo - Rua da Reitoria, 374 Cidade Universitária - CEP: 05508-220 Butantã (SP), Brazil

E-mail: kaiokeomma@usp.br

Declaration of interests: Nothing to declare.

This is an open access article distributed under the terms of the Creative Commons Attribution License.

(C) 2021 The authors

\title{
Adherence in Brazil is
} phenomenal, but early treatment with chloroquine and hydroxychloroquine does not reduce mortality from COVID-19

\author{
Kaio Keomma ${ }^{1}$, Alessandro Leite Cavalcanti ${ }^{2}$ \\ ${ }^{1}$ Faculdade de Saúde Pública, Universidade de São Paulo (FSP/USP) - São Paulo (SP), Brazil \\ Universidade Estadual da Paraíba (UEPB) - Campina Grande (PB), Brazil
}

Early treatment for COVID-19 with chloroquine (CQ) or hydroxychloroquine (HCQ) does not reduce mortality and probably does not reduce the number of cases that progress to mechanical ventilation but may triple adverse effects ${ }^{1}$. However, the adherence to this intervention and the continuity of studies on this topic, especially in Brazil, is phenomenal, considering that: 1) its mechanism today is considered implausible, and 2) there is no high-level evidence to suggest its effectiveness.

A proposition is plausible when it is consistent with existing knowledge in a given area of science, making it rationally defensible. Being plausible is not a determining condition for something to be true. There are other criteria to judge a recommendation and especially to establish a relationship of causation ${ }^{2}$. Still, usually what is true or causal is plausible.

Evidence can be understood as the best scientific knowledge available, achieved by different means, namely techniques and procedures, which are called methods. As there is not a unique Science, but different areas of science, researchers from each one of them more or less validate and often rank different methods of knowledge production. Based on certain assumptions, a researcher admits or contests evidence. This is what makes science comparable.

In Health Sciences, there are countless study designs or methods to investigate different propositions. The use of one or the other depends on what is intended to investigate. If you want to analyze the effectiveness of a drug or a set of drugs, or vaccines, or a surgical approach, for example, the most robust methods are the so-called randomized controlled clinical trials.

These studies work with two groups: in one of them the intervention of interest is applied and in the other, a placebo or conservative treatment is administered. The participant is randomly allocated to one of these groups and must not know which one beforehand. Ideally, the evaluator is also unaware of the information. This is a double-blind study, a procedure that avoids the manipulation of results. To compare the two groups, there is a statistical sophistication and a variety of analytical models used. The study can be interrupted at any time, either when the results achieved point to an unforeseen adverse effect that may represent a risk to the participants, or when an adverse effect occurs in greater proportions than what was previously established and considered acceptable ${ }^{3}$. 
Although well-designed clinical trials are essential to support any more complex conduct in health, they are generally expensive, require complex statistical procedures, adherence to strict ethical protocols, and conduction by specialists in Epidemiology, Statistics and Medicine. Furthermore, many interventions to be tested can represent a real risk to the participants' lives. This compels researchers to be parsimonious when proposing such a study. In addition to safety, it is ethically desirable that there is plausibility in what is intended to be tested, and that this leads researchers to the evidence that recommends or not a specific treatment, vaccine, or surgical approach.

This does not mean that a clinical trial study is proposed to confirm a previous hypothesis. However, exposing thousands of volunteers to risks and mobilizing researchers in methodologically complex initiatives with high costs should propose this design as an ethical, scientific and economically defensible action. In this context, it is understood that not only the indication for the use of CQ or HCQ, but also the proposition of further investigations into the early treatment of COVID-19 should be discouraged. So far, it is not plausible to say that these drugs are effective in preventing the unfavorable evolution of infection with the SARS-Cov-2 coronavirus. There are no high-level studies that even suggest any efficacy and the indications of their adverse effects are increasing.

Perhaps for this reason, some of the objections to the recommendation against using CQ or HCQ to treat COVID-19 are not exactly scientific but are a consequence of a glorification of presumed common sense. Some say that if the idea of early treatment is, so to speak, indefensible from a rational point of view, how can many sustain its use as a public health policy? Others still argue "what about the evidence that suggests that early treatment with these drugs works?". And finally, some cling to what seems to be the last possible argument: doing something is better than not doing anything, right?

Let us begin to question the first objection, according to which it is paradoxical that the treatment of COVID-19 with CQ and HCQ is indefensible and that many doctors still continue to recommend its use. This idea was disseminated at the beginning of the pandemic by some of these same doctors, most of them working in the front line, who believed that, in the midst of the unknown and with the advance of infections, proposing alternatives was better than expecting effective answers from large studies. The apparently satisfactory results obtained in clinical practice supported the hypothesis of the effectiveness of early treatment. Although it has become popular and today, as there is no effective treatment, it continues to be recommended by some doctors, from a scientific point of view, it is absolutely discredited.

In this regard, it is unfortunately necessary to recognize that adopting therapeutic approaches without high-level scientific evidence on their benefits is more common than it seems in the health area. In 2010, for example, according to a study, $37 \%$ of the investigated guidelines on primary prevention strategies in cardiology were considered of low scientific rigor ${ }^{4}$. Despite this fact, many naively believe that the recommendations in documents such as those necessarily represent the best in terms of the recommendation of medical conduct. But, as you can imagine, this is often not true.

The second objection is related to the evidence that treatment with CQ or HCQ supposedly works for COVID-19. However, when it comes to behaviors related to health, it is known that there is evidence for all results. The main question is whether the studies that produced this evidence are sustained after rigorous procedures to evaluate their methods. Blind adherence to recommendations, without a responsible prior evaluation of the studies that support them, should be embarrassing in academia, given that many of them can have a very low scientific level. This is the case with studies that support early treatment. In fact, this is a good area to find instructive examples of scientific biases.

In addition, there are several examples of conflicts between the medical community and high-quality scientific evidence, as a significant number of clinical trials do not prove the expected benefit of a given intervention, even at the cost of millions of dollars and years of study. Thus, the dissatisfaction of prescribers with recommendations against treatments such as CQ or HCQ in COVID-19 is not new. As an example, in 2009, a study recommended not performing routine mammography in women under 50 years old, since the magnitude of the benefit is small and there is no certainty that it exists, at the cost of hundreds of false-positive diagnoses ${ }^{5}$. Although the study argued that the decision should consider the patient's values concerning benefits and harm, not surprisingly, it was received with strong reaction from various segments of society, especially breast cancer specialists and radiologists. Many of them continue to deny its value and disregard its findings.

The third and final objection is more emotional than rational. Therefore, it is distant from the more modern scientific paradigm, according to which a quality health practice must be based on knowledge informed by evidence. They argue that, given the supposed uncertainty of the benefit of early COVID-19 treatment, doing something is better than doing nothing and this itself could justify the ethical principle of beneficence in the use of CQ and HCQ.

Indeed, the best evidence is not always available to guide healthcare professionals. Therefore, in situations of extreme severity, it is necessary to make a decision without adequate scientific support. However, one must insist that currently this is not the case with the use of CQ and HCQ as a therapeutic alternative in the COVID-19 pandemic. The hypothesis of the effectiveness of these drugs in the context of early treatment is not only no longer plausible, as argued before, but there is a strong indication that it actually does not work. Furthermore, the use of this therapeutic strategy can cause harmful damage to those to whom it is administered. 
To mention one last example, in 2016, a series of clinical studies was proposed to evaluate the efficacy of synthetic phosphoethanolamine that had been used by Brazilians for more than two decades without scientific approval as an attempt to treat different types of cancer. Despite an alleged benefit based on the subjective experience of different patients, the main studies were discouraged because they did not show positive clinical results. There was no evidence for efficacy and safety, as there is no evidence today. In addition, ANVISA, the Brazilian regulatory agency, continues to not recommend its use. It seems that the big difference between the case mentioned and the use of CQ and HCQ to treat COVID-19 is that a considerable part of physicians now joins ordinary people in a great appeal for something that has no efficacy.

As stated before, the number of adherents, mainly in Brazil, to the so-called early treatment of COVID-19 with CQ and HCQ and the continuity of studies in this regard is phenomenal, considering the current total lack of plausibility, absence of high-level evidence proving its efficacy and the countless adverse events that have been reported. Given the current state of the art, defending it means being unscientific, and demonstrates contempt for ethics and irresponsibility with resources that could be undertaken in other more promising research areas. It is irresponsible to defend it as a policy of a public health system that represents the only opportunity to provide care for millions of citizens.

\section{REFERENCES}

1. Singh B, Ryan H, Kredo T, Chaplin M, Fletcher T. Chloroquine or hydroxychloroquine for prevention and treatment of COVID-19. Cochrane Database Syst Rev. 2021;2(2):CD013587. https://doi.org/10.1002/14651858.CD013587.pub2

2. Hill $A B$. The Environment and disease: association or causation? Proc R Soc Med. 1965;58(5):295-300.

3. Escosteguy CC. Estudos de intervenção. In: Medronho AM, Carvalho DM, Bloch KV, Luiz RR, Werneck GL. Epidemiologia. São Paulo: Atheneu, 2002; p.151-61.
4. Ferket BS, Colkesen EB, Visser JJ, Spronk S, Kraaijenhagen RA, Steyerberg EW, et al. Systematic review of guidelines on cardiovascular risk assessment which recommendations should clinicians follow for a cardiovascular health check? Arch Intern Med. 2010;170(1):27-40. https://doi.org/10.1001/archinternmed.2009.434

5. Calonge N, Petitti DB, DeWitt TG, Dietrich AJ, Gregory KD, Grossman D, et al. Screening for breast cancer: U.S. Preventive Services Task Force recommendation statement. Ann Intern Med. 2009;151(10):716-26.

https://doi.org/10.7326/0003-4819-151-10-200911170-00008 